\title{
Incorpo rating Stakeholder enfranchisement, Risks, Gains, and AI decisions in AI Governance Framework
}

\author{
Anna Sidorova \\ University of North Texas \\ anna.sidorova@unt.edu
}

\author{
Kashif Saeed \\ University of North Texas \\ kashif.saeed@unt.edu
}

\begin{abstract}
The emergence of AI-enabled applications has drawn attention to the need for AI governance. This essay builds on organizational governance literature and proposes a framework for developing organizational governance structures. Following a call to incorporate all stakeholders in governance [1], the framework considers the interests of all organizational stakeholder groups. In addition, it delineates five types of AI-related organizational decisions, which have the potential to significantly impact stakeholder interests. Furthermore, the framework considers four distinct outcomes and byproducts of AI which may impact the distribution of stakeholder benefits and risks. These need to be specifically addressed by organizational AI governance structures. We contend that the details furnished by the framework pave the way for future research on AIgovernance, adaptation in an AI-driven organization, and AI-related legal framework development.
\end{abstract}

\section{Introduction}

"All stakeholders must participate in the gains and loss es of any particular situation."

- Chris tine Lagarde, Pres ident European Central Bank

Every day, organizations make decisions that are related to the strategy, investments, development, and use of AI, and there are millions of operational decisions that are made daily with the help of AI. But how can an organization ensure that in making these decisions, it protects the interests and investments of its key stakeholders? This organizational AI govemance question has received relatively little attention to date. Technical AI research, for the most part, focuses on the development and use of AI solutions for specific business problems [2]-[4]. Sociotechnical AI research often deals with the idealistic as pects of AI such as the ability of a machine to develop human level intelligence and the roles such machines would play in the future [5]. Extant AI governance discourse focuses primarily on the design principles for ethical and trustworthy AI and government or public sector approaches to AI regulations [6], with little connection to corporate and organizational governance literature, or even IT governance. However, in practice, decisions about and made by AI are largely made in accordance to the combination and organizational and IT govemance rules. We argue that the introduction of AI into organizational fabric calls for the revision of those rules to ensure that key stakeholder interests are accounted for.

Corporate governance discussions in management literature are based on agency theory [7], [8], which calls for the enfranchisement of all stakeholders including vendors, suppliers, customers, employees, etc. Notably, the management literature, to date, has focused on two stakeholders: business management and shareholders [1]. The governance discourse in IT, which, for the most part, draws from the management governance literature, dichotomizes the stakeholders into IT management and business management [9][12]. Yet, we contend that consistent with the recent calls in AMR for a theory of stakeholder governance [1], and the dis cussions of stakeholder enfranchisement and claimancy rights [13], AI governance needs to consider interests of all relevant stakeholder groups.

This theoretical essay considers the externalities created by AI systems through the lens of organizational governance literature and IT governance discourse to propose an AI framework for organizational AI governance. With the proposed framework, we seek to address the following research questions: A) What is the goal of organizational AI governance? B) What is the scope of organizational AI governance? C) How should organizational AI governance reflect the distinct characteristics of AI technology? This essay offers a typology of AI-related decisions based on IT management literature and discusses how the key distinguishing characteris tics of AI translate into new benefits, as well as risks, for organizational stakeholders. Finally, recommendations on the AI governance layers are dis cussed. 


\section{Theore tical foundations}

In this section, we discuss the corporate govemance literature with emphasis on the calls for a theory of stakeholder governance [1] and a governance adaptation perspective centered on stakeholders enfranchisement, claimancy rights, and residual rights of control [13]. Next, we discuss IT governance drawing from the overarching corporate governance literature. We discuss the generalization of stakeholders in IT governance, which describes the need of a framework for AI governance.

\subsection{The role of $A I$ in organizations}

AI is an umbrella term for technological solutions that incorporate capabilities commonly associated with human cognitive functions, such as learning, perception, communication and reas oning [14], [15]. Today's AI solutions powered by statistical machine learning algorithms are capable of learning from data and processing natural language and visual input. These solutions capable of replacing humans workers, supporting human work or even collaborating with human actors in a variety of contexts are becoming a critical part of human-AI labor platforms [16]. Depending on the applications domain, AI technology can enhance existing organizational capabilities by integrating with existing products or processes, or prove to be a capability-destroying technological discontinuity [17], [18]. To date, much of the AI research focused on the technical side of AI or on the applications of AI to specific tasks and contexts, but calls have been made for the study of AI interactions with the organizational and social structures [19]. Corporate governance and IT governance literature provide a theoretical foundation for understanding the interaction of AI with organizational governances tructures.

\subsection{Corporate governance and stakeholders}

Corporate governance work in the management literature is based on theagency theory [7], [8]. Till date, most work on corporate governance focuses on how to resolve potential conflicts of interest between the senior management of the firm and its stakeholders [1]. This work has widely been accepted and implemented by many firms across the globe [20] and has laid the foundation for the mechanis mofgovernance in practice [21]. However, despite Jensen and Meckling's [8] explanation of stakeholders (that include employees, suppliers, customers, creditors, etc.), much of the discussion on corporate governance has been limited to the firm's management and the shareholders [1].

An AMR editorial by Amis et al. (2020) discusses the need for a theory of stakeholder governance [1]. This call for a theory of stakeholder governance is based on the conceptualization that the value created by a firm goes beyond the shareholders. If the non-shareholder stakeholders do not provide profit-generating resources, there will not be profit to distribute to the shareholders [22].

The need for incorporating all stakeholders was underscored in a recent AMR article by Klein et al.[13]. According to Klein, key questions of governance are those of stakeholder enfranchisement, i.e. "who is in, who is out" of organizational decision-making, and claimancy rights (i.e. how the value created by an organization is distributed among its stakeholders). More specifically, organizational governance seeks to answer the following questions: who is involved in decision making, how the benefits (the value created by the organization) are distributed among stakeholder groups, and who bears the risks associated with organizational activity [13]. However, the discussion of stakeholders has largely stayed with shareholders and has not incorporated the larger stakeholders [1]. In the rest of the paper, we will focus on outlining how we can incorporate internal and external IT and business stakeholders in the discussion on formulating an AI governance framework. Moreover, we will focus on answering the questions highlighted by Klein at al. (2019) as a guiding principle for creating AI govemance framework.

\subsection{IT governance}

The use of the term governance in the IT domain has focused more on the participation in decisionmaking, rather than the value of the re-distribution of risk. IT governance is defined as a "framework for decision rights and accountabilities to encourage des irable behavior in the use of IT" [23]. Furthermore, Weill suggests that IT governance is not about what specific decisions are, who makes each type ofdecision (a decision right), who has input to a decision (an input right), and how these people or groups are held accountable [23]. This definition is in line with Klein's explanation of governance structures as a set of rules that define rights and accountabilities [13]. This definition is also in line with an earlier definition of governance by Boynton etal. (1992) which suggests that IT governance is not concerned about the distribution of resources, rather the distribution of management responsibilities [9].

A literary review of IT governance in IS research suggests that the goal of IT governance is to administer 
and guide an organization's IT-related actions and decisions to achieve the desired outcomes [11]. Earlier dis cussions on IT governance, among other is sues, have highlighted the importance of IT structure, that is, the level of centralization of IT in the organization [10], [12], federal mode of governance [24], and the drivers of IT governance structure [25], [26]. While many researchers focused on the structural as pect of IT, other researchers suggested that governing is a negotiated coordinating process that evolves over time, along with the governance structures [27]. Other researchers like Kude et al. (2015) used the RBV to discuss how IT governance capabilities (structures, processes, relational mechanisms ) lead to business synergies [28].

Prior IS res earch has emphasized the importance of engagement between IT and business management [29]. Huang et al. (2010) explored influencing the effectiveness of IT governance through steering committees, suggesting that incorporating executivelevel stakeholders in the committee oriented the committee goals to be broader and longer-term, whereas incorporating operational/functional-level executives in the committee resulted in narrower short-term orientation [11]. Although the same general IT governance framework was implemented through the steering committee, the outcome differed by bringing stakeholders from different levels in organization. Despite Huang et al. (2010) discussion on stakeholder engagement, the IT governance discussions in IS research, for the most part, stay at a macro-level. This provides a vanilla framework for IT governance. The macro-level IT governance discussions focus on IT centralization [9], [10], [12], IT alignment [11], [30], IT strategy[25], IT executive decision making [26], [31], etc.

\subsection{Why we need a separate governance framework for AI?}

The discussion thus far highlighted the need for incorporating stakeholders in governance discussion [1] and the need for the IS literature to develop technologyspecific governance frameworks for different technology sub-domains in IT. Next, we dis cuss how AI is different from other technologies.

Theoretical definitions of artificial intelligence allude the ability of machines to exhibit a human level of intelligence or even conscience, and are notbound by any specific technology or algorithmic solutions [14]. At the same time businesses adopt a practical notion of AI which encompasses IT solutions through the use of machine learning (ML), in conjunction with optimization-based and rule-based algorithms for insight generation, decision support and decisionmaking [32], [33]. In this section we will rely on this practical, technology bound definition, as it is sufficiently tangible to help identify the distinguishing characteristics of AI-enabled solutions and develop an AI governance framework.

Whether used for insight generation, decision support, or decision au tomation, ML-based AI s olutions share the following common characteristics which are relevant to this discussion: (1) their development and maintenance requires large volumes of machine readable data, (2) they involve the creation of new knowledge in the form of a trained model, i.e. knowledge not directly consumable or verifiable by humans, (3) they are capable of producing autonomous or semi-autonomous outputs, such as insight, recommendations, decisions, actions, or artifacts, and (4) once deployed, they enable insight generation and decision automation at a significantly faster rate than human decision making (and at a fraction of the cost per decision). These characteristics have implications for how value is created by an organization and the contributions made by different stakeholder groups to value creation.

These distinct characteris tics of AI require lead to the emergence of new values sources (benefits) and new risks, thus requiring the renegotiation of existing stakeholder arrangements, and often the engagement of new stakeholder groups in the decision-making process.

\section{AI governance frame work}

Building on the corporate governance theory, we conceptualize AI governance as a set of structures that ensure that relevant organizational stakeholders continue to work together and contribute their resources (money, time, effort, data). Said resources are necessary for the achievement of organizational goals when $\mathrm{AI}$ is introduced into organizational socio-technical ecosystem. In devising AI governance, it is critical toensure that all relevant stakeholder groups are accounted for. Regarding traditional firms, existing stakeholder groups include shareholders, employees, customers, and suppliers [8]. New stakeholder groups may include AI developers and vendors, AI solution and data providers, as well as cus tomers in AI-enabled markets.

As AI is introduced, it affects all three components that are traditionally addressed by governance: (1) how decisions are made and how different stakeholder groups are engaged in decision making, (2) how value (benefits) associated with AI is dis tributed, and (3) who bears the risks associated with AI. We contend that the goal of organizational AI governance is to providing answers to these three questions.

Consistent with this goal, the proposed AI governance framework provides guidance about the relevant risks and benefits that are distinct for AI- 
enabled systems, relevant decision types, relevant stakeholder groups, and the building blocks of $\mathrm{AI}$ governance structure.

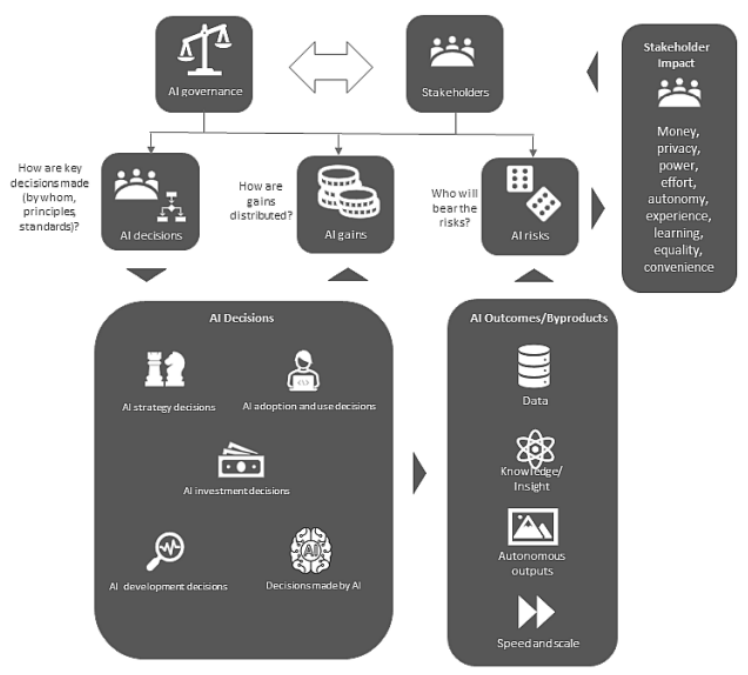

Figure 1. Proposed Al governance framework

\subsection{Relevant stakeholder groups}

Identification of relevant stakeholders is the first step in defining the scope of AI governance. For determining the relevant stakeholders for AI, we follow the guidelines in Jensen and Meckling's recommendations from the theory of the firm that stakeholders should include employees, suppliers, customers, creditors, vendors, etc. [8]. For the org anization and understanding perspective, we propose two dichotomies of the stakeholders: internal vs. external stakeholders, and stakeholders associated with upstream processes vs. downstream processes. These dichotomies are not mutually exclusive, rather they provide two different lenses to view and acknowledge the AI stakeholders in the organization. We explain these dichotomies next.

The external stakeholders include the firm shareholders, customers, suppliers, and vendors (including suppliers of external data and IT vendors). Many of the AI applications deal with customers' data whether it be recommending better products and services to the customers, or enhancing customers' experience using AI, customers are the focal point of most, if not all, AI solutions. Customers gain better experience, better service, and better products, while ris king their privacy. However, customers' perceptions vary in terms of use of their data by the organization; some customers value the gains, while others are concerned about therisks. By making AI a part of their relationship with a customer, organizations can gain more business from the existing customers, earn trust, gain new customers through the word of mouth, and reduce customer churn. On the other side, the organization risks loss of revenue, customer loyalty and trust if the customers' expectation about privacy and ethical use of data are not met.

AI implementations often use external data and IT vendors. Data vendors are third-party org anizations that sell secondary data, which is often used for augmenting the internal data sources or for comparis ons with the market or competitors. IT vendors and suppliers are used to provide software or resources for AI implementations. In these relationships with the IT vendors or suppliers, the organization gains access to readily us able resources in the formofdata, software, or skills. However, the organization risks the dis closure of competitive strategy, extensive cost, reliance on external resources, and delay in building in-house capabilities.

The internal stakeholders include focal process participants, including employees and decision makers, the IT organization, and the data management group that is often a part of the IT organization. The data management group houses and maintains the corporate data resources, which are used by AI/ML algorithms. Moreover, a byproduct of AI is the creation of insights and maturity of exis ting data, which is also managed by the data management group. In addition to providingthe data repositories, IT can offer expertise in application integration, software development, IT in frastructure and architecture support for building scalable AI solutions, and lastly, project management expertise in executing the AI projects.

The second dichotomy views the stakeholders from the upstream and downstream processes pers pective. Ups tream processes are those processes that create data that is used for model training and as an input in to model scoring. Downstream processes are those processes that rely on decision made or recommended by AI. For example, an upstream process could include acquiring auxiliary data from customers, suppliers, or vendors and incorporating it in the corporate data repositories. This process may require the resources provided by an IT vendor or internal resources provided by IT. Participants of both upstream and downstream processes are important AI stakeholders, and need to be included in the scope of AI governance.

\subsection{AI-related decisions}

Another lens for defining the scope of AI governance is by the types of decisions included. In organizational context, AI can be viewed as an IT resource which is designed, developed, deployed, and 
used to create value and achieve the strategic objectives of an organization. From the resource-based perspective, relevant decisions include strategy, investment, development and implementation, adoption, and use of AI. However, AI can also be viewed as an actor, possessing a certain degree of autonomy. Therefore, AI governance needs to cover two more types of decisions: decis ions made by $\mathrm{AI}$, as well as AI control and monitoring decisions. We contend that these five decision types, along with the relevant stakeholder groups, define the scope of AI governance.

\subsubsection{AI s trategy decisions}

AI strategy decisions reflect the role AI plays in organizational strategy, and thus define priorities for AI inves tment decisions. AI can be a valuable res ource if it trans lates into customer-valued product enhancements, or cost-cutting productivity improvements. However, to be a source of competitive advantage, organizational AI resources must to be rare, non-imitable and nonsubstitutable [34]. The role of AI strategy is to define (1) the extent to which an organization views AI as a source of competitive advantage, and (2) the specific types of AI investments that would lead to the biggest value creation and without being easily replicated by competitors. Because the field of AI is a highly dynamic one, any one bundle of AI-based resources cannot be a s ource of competitive advantage over a long period of time. Thus, AI strategy also needs to outline how the organization plans to approach the creation of AI dynamic capabilities, i.e. organizational resources that would allow the identification of new opportunities as sociated with AI, devise AI development strategies, and reconfigure existing AI resources to take advantage of new capabilities.

\subsubsection{AI inves tment decisions}

AI investment decisions are concerned with the allocation of organizational resources to the development or acquisition of specific AI capabilities in support specific strategic objectives. AI investment decisions involve the determination of whether a specific AI investment alternative is aligned with the organizational AI strategy, and whether it is economically and technically feasible. AI investment decisions may be related to specific AI projects or AI programs that span multiple projects. They may involve evaluating a business application that includes AI components, vis-à-vis a non-AI alternative, or approving an in-house operational AI development program. Investment decisions rely on estimates of the costs and benefits associated with each investment option, and thus require an accurate estimation of scope. Yet, scope definition is less than straightforward when it comes to AI. Because AI is inherently data-driven, AI investment decisions have critical implications not only for the downstream processes, i.e. the processes using AI outputs, butalso for the up-streamprocesses, i.e. the processes that create the data used by AI for model training and scoring. Therefore, successful AI implementations may include changes to upstream or downstream business processes, and significant investments into data management infras tructure [35]. On the downstream side, the benefits of AI projects can only be realized if AI outputs (recommendations, decisions or automations) are effectively integrated within business applications and are used by humans in an effective manner. Thus, understanding the potential of change management associated with such integration needs to be taken into account when defining the scope of AI implementation projects [36]. Identifying critical stakeholders and engaging them in the investment decisions can help ensure the projects include sufficient incentives for all relevant stakeholder groups to contribute to the project.

\subsubsection{AI design, development and implementation decisions}

AI investment decisions are translated into the scope, costand schedule targets for AI development and implementation projects, and as such, set the stage for AI development decisions. AI development decisions involve the choices that are made by data scientists, ML engineers and application developers involved in the project. What sources of data are reliable? Is data clean enough to be used in real time? How should data transformation pipelines be designed? How should an ML problem be formulated to reflect the business decision needs? How should model performance be evaluated to ensure that business benefits are optimized? What algorithm and how should the system be architected given the business requirements? Athough these decisions appear technical on the surface, they are grounded in the needs of the business and the understanding of underlying business processes. Engaging relevant stakeholders into development decisions can ensure that stakeholder needs are understood and addressed problems are avoided further down the road. This is consistent with the overall IS development literature, which suggests that user engagement in a system's development projects is a critical success factor in system development and implementation projects [37].

\subsubsection{AI adoption and use decisions}

AI outputs may range from decision insight and recommendations to full decision automation. However, the overall success of an AI enabled systemdepends on its ability to integrate with human-led processes, and thus depends on the willingness of humans to use AI, or 
collaborate with it. The questions of IT adoption anduse has occupied a central space in IS res earch, along with related questions of trust, au tonomy, accountability, and privacy. AI adoption and user decisions provide answers to questions such as: Should AI be used? Who should use AI? How should AIbe used? How much should AI be trus ted? Es tablishing rules for making such decisions falls within the scope of AI governance. Although not explicitly framed as such, many of the responsible/trustworthy/ethical AI frameworks have focused on the AI adoption and use decisions, and how they are influenced by AI development decisions. For example, the principle of autonomy considered a cornerstone of responsible AI suggests that humans should be able to make informed decision about the use of AI. But what does it mean in organizational contex? Would including representatives of a stakeholder group into making an adoption decision on behalf of all group members be enough for satisfying the autonomy principle? AI adoption and use decisions also include defining what constitutes the appropriate and effective use of AI. Does it mean full trust in AI decisions, or the "hands on the wheel" approach? Having an answer to this process may be helpful in informing not only AI development, but also AI investment decisions.

\subsubsection{Decisions made by AI}

At the organizational level, $\mathrm{AI}$ is adopted to support or automate business decisions. AI decision logic is not defined a priori, but is learned from data and adjusted based on environmental responses [15]. Furthermore, such logic is expressed in the form of complex mathematical expressions and is not easily understood by humans. This gives AI the level of decision autonomy not afforded to other IT, making AI the subject of organizational governance, rather just an object of organizational decisions. Thus, an important aspect of AI governance should deal with defining classes of organizational decisions in which AI can participate, and the degree of such participation. For example, the rules of AI governance could speculate that any decision with value at risk below a certain threshold can be made by AI without any human oversight. It may also speculate that certain level of explainability be required for AI to be used for certain types ofdecisions, such as those affecting the well-being of human stakeholders. Importantly, many organizational decisions are made collaboratively and involve the renegotiation of stakeholder participation in value and risk sharing. When such decisions are made by AI, stakeholders' ability to defend their interest in the decisions is lost, resulting in potential disenfranchis ement of important stakeholder groups.
Recognizing the negotiations as pect of organizational decision making, creating adequate appeal and grievances procedures for decisions made by AI can help maintain stakeholder engagement, but also improve the AI-based decis ion making in the long run.

These five decision types outlined here are interrelated, and together they constitute the scope of organizational AI governance.

\subsection{AI Risks and benefits}

To ensure that organizational AI governance reflects distinct characteris tics of AI technology, the gains and ris ks of AIneed to be considered in relation to its various outcomes (and byproducts). These include the vast volumes of data needed to support AI, the migration of org anizational knowledge froma human-readable to machine-readable format, the actual outputs produced by AI, and the scale and speed at which these outputs can be produced.

\subsubsection{AI data dependence and the need for dynamic renegotiation}

The dependence of AI on data significantly increases the relative value of machine-readable data vis-à-vis other resources in an organization. Not only does it make data a necessary input into the value creation process, but it also makes it a potentially valuable output, that can be monetized [38]. At the same time, creation of AI-usable data is associated with perceived and actual costs for stakeholders. Such costs range from additional efforts associated with data disclosure, to investments in converting sensing technologies or the conversion of human-readable data into a machine-readable format, to privacy loss. This creates pressure for the re-negotiation of claimancy rights, which are "therules on the distribution of jointly created value" [13]. Stakeholders involved in the production of data can expect higher levels of compensation to ensure the congruence between res ource provisions and value appropriation. Secondly, new rules need to be established regarding the appropriation of the value created in the formof data. In the abs ence of such rules, the value created through data would be appropriated by stakeholder groups with residual claimancy rights, such as firm shareholders. In the abs ence of appropriate adjustments to the claimancy rules, stakeholders involved in data production may resist participation in the data creation, by refusing to cons ent to corporate use of personal data, participating in organizational politics or social movements, or simply failing to put additional effort necessary for producing high quality AI-usable data. As new AI systems come on board, the value associated with 
different data resources changes, creating the need to governance mechanisms that would allow dynamic renegotiation of claimancy rights over data resources. Such renegotiation of claimancy rights requires stakeholder enfranchisement mechanisms would include organizational stakeholders involved in the production of data in the AI-related decisions.

\subsubsection{Machine-readable knowledge}

A key characteristic ofmodern AI is the shift from human-centered to machine-centered knowledge production. In a broad sense, knowledge can be thought of as a collection of assertions about the world that we believe to be true, and thus base our actions on them Traditionally, knowledgecreation and transfer has been viewed as a strictly human phenomena, related to cognition and human information processing. Not surprisingly, machine learning, i.e. the ability of a computer to create knowledge has been considered a holy grail of AI, along with other knowledge related subfields of AI such as automated reasoning and knowledge representation [39]. Early forms of AI, such as expert systems, relied on the codification of human knowledge, i.e. the knowledge that was primarily created for human consumption and then adapted for use by AI. In contrast, ML-based AI is able to create knowledge in the form of machine-readable models. As such, the knowledge is created primarily for machine consumption, and is only suitable for human consumption with the help of secondary mechanisms, such as explanation algorithms. This shift has important implications for organizational value creation and appropriation, and thus may have significant impact on claimancy rights and stakeholder enfranchisement. Like data, knowledge is an important resource used in organizational value creation, but also has intrinsic value to the extent that it can be internalized by a stakeholder and then applied in a different situation. As such, knowledge can be viewed as a contribution by a stakeholder, but also as a valuable asset, along with other types of intellectual property. As machine knowledge becomes a viable substitute for human knowledge, organizational stakeholders may perceive their knowledge contribution as less valuable, invest less in knowledge acquisition, and agree to a reduction in claimancy rights by accepting lower compensation or become disenfranchised by withdrawing from organizational decision processes. On the other hand, the shift to machine knowledge can make knowledge acquisition more difficult for humans, thus making it more difficult to appropriate value through learning on the job. Lack of machine-to-human knowledge trans ferability creates the risk of dis enfranchisement on the part of human stakeholders, as they may believe that they do not have sufficient knowledge to contribute to organizationaldecisions.

Another implication of machine knowledge is that it leads to the creation of AI-generated IP. Although the AI-generated painting that fetched $\$ 469 \mathrm{~K}$ at Christy auction in 2019 made the international news, relatively little research exists on the topic of AI-generated IP ownership [40]. To date, the share of AI-generated IP is relatively low, but it is bound to increase exponentially as the generative capabilities of AI approach those of humans. For example, OpenAI's NLP service, GPT-3, has been used for the creation of text ranging fromblogs to computer code. To the extent that the content generated by $\mathrm{AI}$ is not particularly valuable, defining the claimancy rights over the value generated by AI may be straightforward. But as the value of AI-generated IP grows, new governance approaches need to be designed and implemented to deal with potential stakeholder conflicts.

\subsubsection{Autonomous outputs of AI}

Enabling machines to make decisions and select a course of action autonomously, based on the state of the environment, is at the core of the AI development efforts. Today, AI can make a variety of simple decisions with the degree of accuracy similar to or above that achieved by human decision makers. Most of us are happy to delegate mundane decisions such as spam filtering or finding the optimal route to a client's location to algorithms. Algorithms are increas ingly deployed in organizations to make a variety of decisions, usually of repetitive and mundane kind, such as checking for manufacturing defects, placing inventory orders, or recommending the best product based on the customer characteristics. The transfer of decision-making responsibilities to AI creates benefits and carries risks, and the AI governance needs to provide guidance on the allocation of such risks and benefits. An example, which might be a bit extreme in terms of consequences, is the us e of opaque AI systems in clinical decision making. In this scenario, an AI system would accept information about a patient and make recommendations for the patient's care to the clinician [41]. The process speeds up the clinician's time for case evaluation and diagnosis, which benefits the hos pital adminis tration in terms of gains [42]. However, in the case of a loss, which could be as severe as the loss of a patient's life due to incorrect treatment, there are many concerns about opacity, responsibility, and accountability among the key stakeholders: hospital administration, hospital IT, vendor for AI solution, hos pital data owners, and clinician [42]. 


\subsubsection{The scale and speed ass ociated with AI-based decisions}

Apart from the benefits and risks associated with individual AI-based decision, the ability of AI to make decisions at a speed and scale that cannot be achieved by human decision makers carries a separate set of benefits and risks for different stakeholder groups. Faster decisions can translate to faster service, and thus create value for the customer. They can also result in devaluing the contributions of exis ting human decision makers, resulting in the need for the renegotiation of stakeholder rights.

\section{Dis cus sion, implications, \& future research}

The AI framework defines the goal of AI governance in terms of the questions about stakeholder enfranchisement and distribution of value and risks associate with $\mathrm{AI}$, and outlines the scope of $\mathrm{AI}$ governance in terms of relevant stakeholder groups and decision types. Furthermore, the framework draws attention to fourdistinct characteris tics of AI that must be addressed by AI governance structures. But how can AI goals be achieved in practice? Insights about building blocks of a governance structure can be gleaned from the regulation theory and AI govemance frameworks in the public domain [43], which points to policies, processes and collaborations as critical building blocks of AI governance. Using the organizational AI framework presented here and combining it with the framework furnished in W irtz et al. [43], processes can be defined for AI-related risk management, framing, and risk-benefit analysis for AI at the corporate as well as project level. Similarly, social, ethical, organizational, and IT policies can be defined to address the risks related to AI. Moreover, collaboration bodies such as stakeholder committees, and regulation and governance committees can be designed at IT, project, departmental, and corporate level. The goal of having these committees at different levels in the organization is to keep checks and balances and to avoid unforeseen risks related to social, ethical, and legal challenges for the organization and its stakeholders.

Moreover, the AI governance framework and its components discussed in this paper provides the grounding for future AI res earch studies. For example, this research framework could be used to inform the organizational actors to facilitate adaptation and the pathways available for organizational actors should the external and institutional environment for the organization changes. External environment (EE), generally includes technology, demand, and institutional shocks that affect the firm's behavior and performance, whereas institutional environment (IE) refers to the specific formal and informal legal, political, and social structures and processes in the EE that frame organizational governance structures [13]. More specifically, the AI governance framework pres ented in this paper informs the enfranchised stakeholders to renegotiate the risks, gains, and claimancy rights when the organization experience changes in the IE and EE. Furthermore, the framework provides the means of analyzing the impact of AI as an EE as well as an IE agent. Lastly, The lack of opacity, accountability, and responsibility in healthcare for the use or AI systems was recently highlighted by Helen Smith [42]. We contend that the framework furnished in this paper coupled with the regulation framework by Wirtz et al. [43] can help regulate different accountability, opacity, and responsibility scenarios that will provide the basis for legal frameworks for AI systems across distinct domains and sectors.

The goal of this article, more specifically the AI governance framework, is to provide a holistic view of AI in the organizational context that can be used to inform IS researchers as well as practice. The AI governance framework highlights several directions for building testable hypotheses regarding specific AIrelated decision types and AI risks and benefits. Such hypotheses are expected to draw on the theoretical basis that are most closely related to the decision domain. For example, AI adoption and use decision hypotheses are expected to draw on the extensive technology adoption and trust literature, whereas hypotheses related to AI strategy decisions are expected to draw on strategic management theories. Last, but not least, evaluating and comparing the AI risks, gains, stakeholders, and AI decisions across task substitution (AI substitute humans), task augmentation (AI and humans complement each other), and task assemblage (AI and humans function as a unit) [5] provides unique insights for future research.

\section{Conclusion}

Drawing on organizational governance literature, the framework defines the goal of organizational AI governance as providing answers to three questions: Who is involved in decis ion making about AI? How are the benefits of AI distributed among organizational stakeholders? Who bears the risks associated with AI? The framework further outlines the scope of AI governance in relation to key stakeholder groups and five relevant decision types: AI strategy, AI inves tments, AI development, AI adoption and use and decisions made by AI. It further delineates therisks and benefits associated with $\mathrm{AI}$ in relation to four distinct characteristics of AI technology: reliance on data, 
machine knowledge, autonomous decisions and scale and scope. We hope that the proposed framework can serve as a starting point in defining processes, policies, and collaboration bodies that can furnish the govemance and AI regulatory implementation at an organizational level. We also contend that AI framework presented in this paper provides the foundational bas is for redistribution and renegotiations of risks, benefits, enfranchisements, and claimancy rights to facilitate organizational adaptation. Lastly, we contend that the AI framework presented in this paper provides the grounding and future research agenda for creating legal frameworks for mitigating opacity, accountability, and responsibility issues related to AI-drive decision making.

This study contributes to the IS literature and informs future research on AI in many ways. One, we addressed the dichotomous categorization of stakeholders in IS research by providing a framework for AI that incorporates enfranchised stakeholders, risks, benefits, and decisions. This will pave the way for more granular governance dis cussions in IS res earch for other sub-domains of IT. Two, the framework for AI that lays out the key components of AI govemance provides grounding for future research in many directions. For example, the details furnished in the papercan be combined with the processes, policies, and collaboration bodies discussed by Wirtz et al. [43] to inform governance structures for public, private, and public-private partnerships. Similarly, our framework of AI components can provide the foundational bas is for redistribution and renegotiations of risks, benefits, enfranchisements, and claimancy rights to facilitate organizational adaptation [13]. Lastly, our framework can provide the foundational basis for legal frameworks for opacity, accountability, and responsibility [42] for AI system use in different public and private organizations.

\section{References}

[1] J. Amis, J. Barney, J. T. Mahoney, and H. Wang, "Why we need a theory of stakeholder governanceand why this is a hard problem," Academy of Management Review, vol. 45, no. 3. pp. 499-503, 2020, doi: 10.5465/AMR.2020.0181.

[2] F. Jiang et al., "Artificial intelligence in healthcare: past, present and future," svn.bmj.com, doi: 10.1136/svn-2017-000101.

[3] A. M. U. D. Khanday, S. T. Rabani, Q. R. Khan, N. Rouf, and M. Mohi Ud Din, "Machine learning based approaches for detecting COVID-19 using clinical text data," Int. J. Inf. Technol., vol. 12, no. 3, pp. 731-739, Sep. 2020, doi: 10.1007/s41870020-00495-9.

[4] S. Schanke, G. Burtch, and G. Ray, "Estimating the
Impact of 'Humanizing' Customer Service Chatbots,"Inf. Syst. Res., p. isre.2021.1015, 2021, doi: $10.1287 /$ isre.2021.1015.

[5] A. Rai, P. Constantinides, and S. Sarker, "Next Generation Digital Platforms:: T oward Human-AI Hybrids," MIS Q., vol. 43, no. 1, pp. iii-ix, Mar. 2019, Accessed: Jun. 05, 2021. [Online]. Available: https://www.research.manchester.ac.uk/portal/en/pu blications/next-generation-digitalplatforms(f8e763a6-7cec-4522-9ff1ee3758911274).html.

[6] M. Kuziemski and G. Misuraca, "AI governance in the public sector: Three tales from the frontiers of aut omated decision-making in democratic settings," Telecomm. Policy, vol. 44, no. 6, p. 101976, Jul. 2020, doi: 10.1016/j.telpol.2020.101976.

[7] H. Alchian, A. A., \& Demsetz, "Production, information costs, and economic organization," $\mathrm{Am}$. Econ. Rev., vol. 62, no. 5, pp. 777-795, 1972.

[8] M. C. Jensen and W. H. Meckling, "Theory of the firm: Managerial behavior, agency costs and ownership structure," J. financ. econ., vol. 3, no. 4, pp. 305-360, 1976.

[9] A. C. Boynton, G. C. Jacobs, and R. W. Zmud, "Managing information technology: just whose responsibility is it?," MIT Sloan Manag. Rev., pp. 138, 1992.

[10] R. W. Zmud, A. C. Boynton, and G. C. Jacobs, “The information economy: A new perspective for effective information systems management," $A C M$ SIGMIS Database, vol. 18, no. 1, pp. 17-23, Sep. 1986, doi: 10.1145/20724.20726.

[11] R. Huang, R. W. Zmud, and R. L. Price, "Influencing the effectiveness of IT governance practices through steering committees and communication policies," Eur. J. Inf. Syst., vol. 19, no. 3, pp. 288-302, 2010, doi: 10.1057/ejis.2010.16.

[12] R. F. Jenkins, J. M. and Santos, "Centralization vs. decentralization of data processing functions," Econ. Inf. Process., vol. 2, pp. 62-67, 1982.

[13] P. G. Klein, J. T. Mahoney, A. M. McGahan, and C. N. Pitelis, "Organizational governance adaptation: Who is in, who is out, and who gets what," Acad. Manag. Rev., vol. 44, no. 1, pp. 6-27, 2019, doi: 10.5465/amr.2014.0459.

[14] J. McCarthy, M. L. Minsky, N. Rochester, and C. E. Shannon, "A proposal for the Dartmouth summer research project on artificial intelligence," AI Mag., vol. 27, no. 4, pp. 12-14, 2006.

[15] S. Russell and P. Norvig, Artificial intelligence-a modern approach. Englewood Cliffs, NJ: Prentice Hall, 2002.

[16] A. Rai, P. Constantinides, S. S.-M. Quarterly, and U. 2019, "Editor'Scomments: next-generation digital platforms: toward human-AI hybrids," MIS Q., vol. 43, no. 1, 2019, Accessed: Jun. 24, 2020. [Online]. Available: https://dl.acm.org/doi/abs/10.5555/3370135.337013 6.

[17] M. L. Tushman and P. Anderson, "T echnological Discontinuities and Organizational Environments," 
Adm. Sci. Q., vol. 31, no. 3, p. 439, 1986, doi: $10.2307 / 2392832$.

[18] A. S. Rao and G. Verweij, "PwC's Global Artificial Intelligence Study: Sizing the prize," 2018.

[19] Q. Nguen, A. Sidorova, and R. Torress, “Artificial Intelligence in Business: A literature review and research agenda," Commun. AIS (accepted Publ., 2021.

[20] R. V. Aguilera and G. Jackson, "The cross-national diversity of corporate governance: Dimensions and determinants," Acad. Manag. Rev., vol. 28, no. 3, pp. 447-465, 2003, doi:

10.5465/AMR.2003.10196772.

[21] O. Williamson, The mechanisms of governance. Oxford University Press, 1996.

[22] J. B. Barney, "Why resource-based theory's model of profit appropriation must incorporate a stakeholder perspective," Strateg. Manag. J., vol. 39, no. 13, pp. 3305-3325, Dec. 2018, doi: $10.1002 / \mathrm{smj} .2949$.

[23] J. W. Weill, P., \& Ross, IT governance: How top performers manage IT decision rights for superior results. 2004.

[24] E. M. Von Simson, "The 'Centrally Decentralized' IS Organization,” Harvard Business Review, vol. 68, no. 4.pp. 158-162, 1990.

[25] C. V Brown and S. L. Magill, "Reconceptualizing the Context-Design Issue for the Information Systems Function," pubsonline.informs.org, vol. 9, no. 2, pp. 176-194, 1998, doi: 10.1287/orsc.9.2.176

[26] V. Sambamurthy and R. W. Zmud, "Arrangements for information technology governance: A theory of multiple contingencies," MIS Q. Manag. Inf. Syst., vol. 23 , no. 2 , pp. 261-290, 1999, doi: $10.2307 / 249754$.

[27] C. K. Williams and E. Karahanna, "Causal explanation in the coordinating process: A critical realist case study of federated it governance structures," MIS Q. Manag. Inf. Syst., vol. 37, no. 3, pp. 933-964, 2013, doi:

10.25300/MISQ/2013/37.3.12.

[28] T. Kude, C. Schmidt, S. Mithas, and A. Heinzl, "Disciplined autonomy and innovation effectiveness: The role of team confidence and task volatility," in 75th Annual Meeting of the Academy of Management, AOM 2015,2015, pp.1615-1620, doi: 10.5465/AMBPP.2015.286.

[29] M. J. Earl, "Experiences in Strategic Information Systems Planning," MIS Q., vol. 17, no. 1, p. 1, 1993, doi: $10.2307 / 249507$.

[30] S. P.-J. Wu, D. Straub, and T.-P. Liang, "HOW INFORMATION TECHNOLOGY GOVERNANCE MECHANISMS AND ST RATEGIC ALIGNMENT IN...: find articles, books, and more.," MIS Q., vol. 39, no. 2, pp. 497$518,2015$.

[31] V. Sambamurthy and R. W. Zmud, "Research
Commentary: The Organizing Logic for an Enterprise's IT Activities in the Digital Era - A Prognosis of Practice and a Call for Research," Inf. Syst. Res., vol. 11, no. 2, pp. 105-114, 2000, doi: 10.1287/isre.11.2.105.11780.

[32] IDC, “IDC Forecasts Strong 12.3\% Growth for AI Market in 2020 Amidst Challenging Circumstances," Idc, 2020.

[33] T. Balakrishnan, M. Chui, B. Hall, and N. Henke, "Global survey: The state of AI in 2020," Mckinsey Anal., no. November, p. 13, 2020.

[34] P. L. Drnevich and D. C. Croson, "Information technology and business-level strategy: Toward an integrated theoretical perspective," MIS Q. Manag. Inf. Syst., vol. 37, no. 2, pp. 483-509, 2013, doi: 10.25300/MISQ/2013/37.2.08

[35] T. Panch, H. Mattie, and L. A. Celi, "The 'inconvenient truth' about AI in healthcare," $n p j$ Digit. Med., vol. 2, no. 1, 2019, doi: 10.1038/s41746-019-0155-4.

[36] A. Mateescu and M. C. Elish, "AI in context: the labor of integrating new technologies," Data Soc. Res. Inst., vol. 30, no. Ml, pp. 1-6, 2018.

[37] M. I. Hwang and R. G. Thorn, "The effect of user engagement on system success: A meta-analytical integration of research findings," Inf. Manag., vol. 35, no. 4, pp. 229-236, Apr. 1999, doi: 10.1016/S0378-7206(98)00092-5.

[38] F. Firouzi, B. Farahani, M. Barzegari, and M. Daneshmand, "AI-Driven Data Monetization: The other Face of Data in IoT-based Smart and Connected Health," IEEE Internet Things J., pp. 11, 2020, doi: 10.1109/jiot.2020.3027971.

[39] S. Robbins, "AI and the path to envelopment: knowledge as a first step towards the responsible regulation and use of AI-powered machines," $A I$ Soc., vol. 35, pp. 391-400, 2020, doi: 10.1007/s00146-019-00891-1.

[40] D. Somaya and L. A. V. R. Varshney, "Ownership Dilemmas in an Age of," Issues Sci. Technol., vol. 36, no. 2, pp. 79-85, 2020.

[41] C. Ross and I. Swetlitz, “IBM's Watson supercomputer recommended 'unsafe and incorrect' cancer treatments, internal documents show," STAT+, 2018. https://www.statnews.com/wpcontent/uploads/2018/09/IBMs-Watsonrecommended-unsafe-and-incorrect-cancertreatments-ST AT .pdf (accessed Jun. 13, 2021).

[42] H. Smith, "Clinical AI: opacity, accountability, responsibility and liability," AI Soc., 2020, doi: 10.1007/s00146-020-01019-6.

[43] B. W. Wirtz, J. C. Weyerer, and B. J. Sturm, "The Dark Sides of Artificial Intelligence: An Integrated AI Governance Framework for Public

Administration," Int. J. Public Adm., vol. 43, no. 9, pp. 818-829, 2020, doi:

10.1080/01900692.2020.1749851. 